\title{
El fuego favorece la invasión de Teline monspessulana (Fabaceae) al aumentar su germinación
}

\author{
Fire promotes Teline monspessulana (Fabaceae) invasion by increasing its germination
}

\author{
RAFAEL A. GARCÍA ${ }^{1,3, ~ *}$, ANÍBAL PAUCHARD ${ }^{1,3}$, LOHENGRIN A. CAVIERES ${ }^{2,3}$, EDUARDO PEÑA $^{1}$ \& \\ MARÍA F. RODRÍGUEZ ${ }^{2,3}$ \\ ${ }^{1}$ Facultad de Ciencias Forestales, Universidad de Concepción, Chile \\ ${ }^{2}$ Departamento de Botánica, Facultad de Ciencias Naturales y Oceanográficas, Universidad de Concepción, Chile \\ ${ }^{3}$ Instituto de Ecología y Biodiversidad (IEB), Chile \\ *Autor correspondiente: ragarcia@udec.cl
}

\begin{abstract}
RESUMEN
A nivel mundial, se ha reportado que las especies invasoras y el fuego pueden interactuar positivamente modificando la dinámica del paisaje y los ciclos ecosistémicos. Este trabajo tiene por objetivo estudiar el efecto de los incendios forestales sobre el banco de semillas de Teline monspessulana (L.) K. Koch (Retamilla) y sus implicancias para la invasión de la especie en la zona mediterránea del centro-sur de Chile. Para cumplir con este objetivo, muestras del banco de semillas fueron sometidas a diferentes tratamientos que simulan las condiciones de un incendio, a través de quemas controladas. Además, para semillas tomadas desde plantas adultas se estimó el rango óptimo de temperaturas a las que ocurre la germinación. Los resultados muestran que después de un incendio forestal la germinación de T. monspessulana aumenta significativamente, siendo el calentamiento de las semillas el factor que gatilla este aumento. Por otro lado, se determinó que las semillas alcanzan su máxima tasa de germinación cuando son precalentadas entre 80 y $120{ }^{\circ} \mathrm{C}$ por 10 minutos, produciendose la muerte del $100 \%$ de las semillas a los $140^{\circ} \mathrm{C}$. Durante un incendio las temperaturas óptimas para la germinación al interior del suelo se alcanzaron cerca de los $2 \mathrm{~cm}$ de profundidad o bien en zonas donde la acumulación de combustible superficial (biomasa) no supera las 5 ton ha ${ }^{-1}$. La pequeña porción del banco de semillas que logra ser estimulada se compensa con la gran cantidad de semillas presente en los bancos que puede superar las 8000 semillas $\mathrm{m}^{-2}$. Este estudio demuestra que el fuego, fenómeno común en zonas mediterráneas y crecientemente frecuente en Chile central, estaría favoreciendo la permanencia de T. monspessulana en áreas con alto grado de intervención antrópica.
\end{abstract}

Palabras clave: Genista, interacción positiva, invasión de plantas.

\begin{abstract}
Worldwide, it has been reported that invasive species and fire can interact positively changing landscape dynamics and ecosystem cycles. This work aims to study the effect of forest fires on the seed bank of Teline monspessulana (L.) K. Koch (French Broom) and its implications for the invasion of the species in the mediterranean area of southcentral Chile. For this aim, soil seedbank samples were subjected to different treatments that simulate fire conditions through controlled burns. In addition, for seeds taken from mature plants the optimal range of temperatures at which germination occurs was determined. The results show that after a wildfire germination of $T$. monspessulana increases significantly. Heating of the seeds was the factor that triggers this increase in germination. Furthermore, it was determined that the seeds reach their maximum germination rate when preheated between 80 and $120{ }^{\circ} \mathrm{C}$ for 10 minutes. At $140{ }^{\circ} \mathrm{C}, 100 \%$ of the seeds die. During a fire, optimal temperatures for germination are reached about $2 \mathrm{~cm}$ in depth within the soil or at the surface in areas where the accumulation of fuel (biomass) does not exceed 5 tons ha ${ }^{-1}$. The small portion of the seed bank that manages to be stimulated is offset by the large seedbank that can exceed 8000 seeds $\mathrm{m}^{-2}$. This study shows that the fire, a common phenomenon in mediterranean areas and increasingly frequent in central Chile, would be promoting the persistence of $T$. monspessulana in areas with high human disturbance.
\end{abstract}

Key words: Genista, plant invasions, positive feedback.

\section{INTRODUCCIÓN}

Los incendios forestales y la proliferación de especies invasoras exóticas pueden interactuar positivamente entre ellos, incrementando los impactos de las especies invasoras en los ecosistemas invadidos (D'Antonio \& Vitousek 1992, Mack \& D’Antonio 1998, D’Antonio 2000, Brooks et al. 2004). Por ejemplo, las invasiones biológicas pueden afectar el régimen del fuego debido a la producción adicional de biomasa, aumentando así la carga de combustible, o 
alterar otros atributos como la inflamabilidad, continuidad y homogeneidad del combustible (Mack \& D’Antonio 1998, Brooks et al. 2004). Adicionalmente, la modificación del régimen del fuego podría acentuar los efectos negativos de las especies invasoras sobre los ecosistemas invadidos al promover la perpetuación de estas especies, las que muchas veces están mejor adaptadas a fuegos frecuentes en comparación a las especies nativas (Brooks et al. 2004).

Existe una serie de mecanismos propuestos para entender la interacción positiva entre fuego e invasión de especies exóticas (Mack \& D’Antonio 1998, D’Antonio 2000, Brooks et al. 2004). Por ejemplo, las especies invasoras pueden acumular bancos de semillas cuya germinación es estimulada por el fuego (i.e. altas temperaturas, humo o combinación de ambos), lo que no ocurre con las especies nativas (e.g., Gómez-González \& Cavieres 2009, Figueroa et al. 2009). Alternativamente, las semillas de las especies invasoras exóticas podrían ser más resistentes al fuego que las nativas (e.g., Alexander \& D'Antonio 2003a).

La constante intervención antrópica de los ecosistemas naturales, unido con el actual escenario de calentamiento global, traen consigo un importante aumento en la cantidad y extensión de los incendios forestales en diversos ecosistemas del mundo (Cramer 2001, Cochrane \& Barber 2008, IPCC 2008). Los ecosistemas con clima tipo mediterráneo, caracterizados por presentar veranos cálidos $\mathrm{y}$ secos e inviernos fríos y lluviosos, son muy propensos a sufrir eventos de fuego debido a que durante la estación seca se incrementa la inflamabilidad del combustible de origen vegetal (di Castri 1991, Keeley \& Fotheringhan 2000). En el caso de Chile, el número de incendios ha aumentado en un $14 \%$ en la última década, concentrándose en la Región del Biobío con una superficie quemada anual promedio de 15430 ha equivalente al $32 \%$ del total nacional (Peña \& Valenzuela 2008, CONAF 2009). A diferencia de lo que ocurre en otros ecosistemas de clima tipo mediterráneo (e.g., California, Sudáfrica, Australia), en Chile central el fuego no es una perturbación natural, siendo causado por acción antrópica en prácticamente la totalidad de los casos (Peña \& Valenzuela 2008).
En Chile central, la interacción fuegoinvasión tendría sus mayores impactos cuando se presente en una comunidad con especies no adaptadas al fuego y la especie invasora provenga de un área donde el fuego sea un agente modelador clave del ecosistema. Se ha visto que el fuego promueve la invasión en ecosistemas con clima tipo mediterráneo. Por ejemplo, Keeley et al. (2003) señalan que bosques de coníferas no incendiados en California están en gran parte libres de especies introducidas, en comparación con la alta presencia de introducidas en las áreas quemadas. Para Chile central, GómezGonzález \& Cavieres (2009) mostraron que en incendios de alta intensidad solo las especies introducidas son capaces de sobrevivir en el banco de semillas, reclutando en forma masiva tras el fuego.

Una especie altamente invasiva en la zona de clima mediterráneo y parte del territorio con clima templado de Chile, es Teline monspessulana (L) K. Koch (Fabaceae; retamilla, retamo, french broom) (Matthei 1995, Pauchard \& Alaback 2002, García et al. 2007). Dado su origen en la cuenca del Mediterráneo, es posible encontrarla también en zonas de similares características y donde los incendios son frecuentes (Australia, Chile, California), así como en otras de clima distinto (Canadá, Nueva Zelanda y otras zonas de EE.UU como Washington y Hawai) (e.g., Johnson 1982, Adams 1991, Bossard 2000, Parsons \& Cuthbertson 2001, Zouhar 2005). Las semillas de $T$. monspessulana poseen una testa dura y pueden permanecer sin germinar durante años, generando abundantes bancos de semillas bajo las plantas madres. Esta capacidad es la que asegura la posterior regeneración masiva de la especie, razón por la cual es considerada una maleza peligrosa de difícil control (Matthei 1995, Alexander \& D'Antonio 2003b, NLWRA 2008). Sin embargo, se ha registrado una muy baja supervivencia de las plantas provenientes del banco de semillas debajo de las formaciones adultas de T. monspessulana (Smith 1994, Waterhouse 1998). De hecho, el retiro del dosel puede ser un requisito previo para una regeneración exitosa.

En Chile, la dinámica de regeneración de $T$. monspessulana y su relación con la ocurrencia de perturbaciones ha sido poco estudiada, pero 
la evidencia empírica demuestra que esta está fuertemente ligada a incendios forestales (Pauchard et al. 2008). En Chile central, aunque el fuego es capaz de consumir completamente las formaciones adultas de $T$. monspessulana, un número importante de sus semillas son capaces de sobrevivir generando una abundante regeneración en la temporada de crecimiento inmediata después del incendio (García et al. 2007). De hecho, la regeneración de T. monspessulana registrada después de un incendio es cerca de 225 veces mayor a la que se encuentra de forma natural bajo individuos adultos de dicha especie en sitios de la cordillera de la costa de Concepción (Pauchard et al. 2008). La mayor regeneración de esta especie postincendio podría estar ligada a una alta resistencia de sus semillas a las altas temperaturas producto de su testa dura y/o a una estimulación de su germinación producto de las altas temperaturas tal como ha sido reportado para otras especies de Fabáceas (Baskin \& Baskin 1998).

En este estudio, se evaluó el efecto del fuego en el banco de semillas de $T$. monspessulana. Para ello, se determinaron las temperaturas que se registran al interior del banco edáfico de semillas durante el fuego, el tamaño del banco de semillas de $T$. monspessulana y la germinación total después de un incendio forestal superficial. Además, se determinó el rango de tolerancia a altas temperaturas de las semillas de $T$. monspessulana para evaluar el efecto de la intensidad de los incendios en el éxito del establecimiento de esta especie.

\section{MÉTODOS}

\section{Especie}

Teline monspessulana es un arbusto leñoso perenne originario de la cuenca del Mediterráneo, cuyos tallos muy ramificados pueden alcanzar alturas de entre 1 y 4 $\mathrm{m}$. Sus hojas son trifolioladas con folíolos de 5 a $15 \mathrm{~mm}$ de largo. Sus flores son amarillas y se reúnen en cortos racimos terminales o axilares. El fruto es una legumbre velluda de 18 a $22 \mathrm{~mm}$ de largo, que contiene de tres a ocho semillas de 2 a $3 \mathrm{~mm}$ de diámetro, redondocuadrangular. Los primeros registros de su presencia en Chile datan de 1847, cuando se le describe como especie ornamental presente en variados puntos del país (Matthei 1995). En la actualidad es posible encontrar poblaciones de T. monspessulana creciendo en Chile continental a lo largo de la Cordillera de la Costa desde la Región de Valparaíso hasta la Región de
Los Ríos (entre los $32^{\circ}$ y $40^{\circ} \mathrm{S}$ ), y el archipiélago Juan Fernández (Matthei 1995). Teline monspessulana es frecuente en el sotobosque de las plantaciones de Pinus radiata D. Don y Eucalyptus globulus Labill, y forma extensos matorrales en áreas abiertas o degradadas, en taludes y claros del bosque. También, es posible encontrarla en orillas de caminos y sitios perturbados en áreas cordilleranas alejadas del valle central.

\section{Área de estudio}

Para el desarrollo de este estudio se obtuvieron muestras del banco de semillas y hojarasca de dos poblaciones de $T$. monspessulana $\left(36^{\circ} 74^{\prime} \mathrm{S}, 73^{\circ} 11^{\prime} \mathrm{O}\right.$ y $\left.36^{\circ} 83^{\prime} \mathrm{S}, 73^{\circ} 03^{\prime} \mathrm{O}\right)$. Además se obtuvo semillas desde las vainas de una tercera población $\left(36^{\circ} 82^{\prime} \mathrm{S}, 73^{\circ} 00^{\prime} \mathrm{O}\right)$. Las poblaciones están ubicadas en la zona costera de la provincia de Concepción, Chile. Todos los sitios presentaban una alta intervención antrópica y estaban cubiertos por poblaciones densas de T. monspessulana en estado adulto. El clima corresponde a mediterráneotemplado cálido con una estación seca corta de cuatro meses. La precipitación promedio alcanza $1100 \mathrm{~mm}$ anuales, de los cuales un 65 a $70 \%$ cae entre los meses de mayo a agosto. La temperatura media anual se encuentra cercana a los $12{ }^{\circ} \mathrm{C}$ y la amplitud térmica anual es de $7.5^{\circ} \mathrm{C}$. El suelo es del tipo granítico, en el cual, la falta de cobertura vegetal lo hace altamente susceptible a la erosión.

\section{Respuesta del banco de semillas al fuego}

En dos de las poblaciones se establecieron seis transectos de $10 \mathrm{~m}$ de largo, separados cada $2 \mathrm{~m}$. En cada transecto se seleccionaron, de forma aleatoria, tres puntos para la toma de muestras de suelo. En cada punto se estableció un área de $20 \times 20 \mathrm{~cm}$ de la que se extrajo toda la hojarasca y material vegetal superficial para la determinación de la carga de combustible (biomasa). En el mismo punto, y con el suelo ya descubierto, se colectó una muestra de suelo de $10 \mathrm{~cm}$ de profundidad por $5 \mathrm{~cm}$ de diámetro. En total, se colectaron por cada una de las dos poblaciones, 18 muestras de hojarasca y 18 muestras de suelo.

Utilizando las muestras de hojarasca y suelo, se testeó el efecto de tres niveles de quemas controladas; una quema con una alta carga de combustible superficial (quema alta), quema con la carga de combustible superficial (quema baja) y sin quema, manteniendo la hojarasca en cada muestra (sin quema).

Las muestras de hojarasca fueron secadas al horno por $48 \mathrm{~h}$ a $80^{\circ} \mathrm{C}$ y pesadas para determinar la carga de combustible en cada población. Las muestras de suelo fueron colocadas en recipientes de aluminio de 11 x 8 x $4 \mathrm{~cm}$, sobre las cuelas se depositó la cantidad de hojarasca correspondiente a cada tratamiento. Según los valores encontrados en este estudio la población con menor carga promedio de combustible alcanzó 5.4 ton $\mathrm{ha}^{-1}(\mathrm{n}=18)$, así en el tratamiento sin quema se mantuvo un equivalente de carga de 5 ton $\mathrm{ha}^{-1}$ sobre cada muestra. Al tratamiento quema baja se agregaron los mismos 5 ton $\mathrm{ha}^{-1}$ de combustible. En el caso de quema alta se duplicó la carga con un equivalente de 10 ton ha-1

En todas las muestras correspondientes a los tratamientos quema alta y quema baja se evaluaron las temperaturas que se registraron al interior del banco 
de semillas durante la quema con termocuplas tipo "K" de $3 \mathrm{~mm}$ de diámetro mineralmente aisladas. En cada muestra se puso una termocupla en la superficie de la muestra y otra a $2 \mathrm{~cm}$ de profundidad, registrándose la temperatura de cada termocupla cada dos minutos durante los diez primeros minutos y cada cinco minutos hasta el final de cada quema. Las cargas de combustible superficial fueron quemadas sobre las muestras de suelo y posteriormente retiradas después que las muestras descendieron a menos de $50{ }^{\circ} \mathrm{C}$ o bien por un tiempo máximo de 90 minutos.

Después de la aplicación de las quemas, todas las muestras fueron llevadas al invernadero de la Facultad de Ciencias Forestales de la Universidad de Concepción, donde fueron regadas en forma automática durante 60 días (febrero-marzo 2008) para provocar la germinación de las semillas. Se efectuó un total de seis repeticiones de cada tratamiento para cada población. Todas las plántulas emergidas en el invernadero fueron contadas y removidas cada dos días durante el periodo de germinación. Una vez concluido el periodo de germinación en invernadero, se tamizaron las muestras de suelo para extraer las semillas no germinadas de $T$. monspessulana, con tamices de 5,2 y $1 \mathrm{~mm}$. En base al número de semillas no germinadas en cada muestra se determinó el porcentaje final de germinación. El número de semillas de $T$. monspessulana presentes en el banco de semillas se obtuvo sumando el número de semillas germinadas más las obtenidas desde el material tamizado.

\section{Temperaturas óptimas para la germinación}

Para determinar el rango óptimo de temperaturas en que ocurre la germinación T. monspessulana, semillas de esta especie fueron expuestas a una combinación de temperaturas y tiempos de exposición en un horno con aire forzado modelo Kottermann 2711. Para ello, se colectaron semillas de T. monspessulana directamente de las vainas de plantas adultas, las que fueron colectadas los primeros días de abril de 2008, al final del periodo de semillación. Las semillas fueron limpiadas en el laboratorio. Se evaluaron 11 temperaturas: $80,90,100,110,120,130,140,150,160$, 170 y $180{ }^{\circ} \mathrm{C}$ y dos tiempos de exposición 5 y 10 minutos, más un tratamiento control en el cual las semillas no fueron precalentadas en horno. Cada tratamiento contó con 80 semillas en total, repartidas en cuatro repeticiones de 20 semillas cada una. Inmediatamente después de la aplicación del tratamiento, las semillas se pusieron a germinar sobre papel filtro en cámaras germinadoras del tipo Jacobsen durante 40 días a una temperatura de $30{ }^{\circ} \mathrm{C}$ por el día y $20{ }^{\circ} \mathrm{C}$ por la noche. El conteo de semillas germinadas se realizó diariamente a partir del séptimo día. Se consideró que la semilla había germinado cuando su radícula presentaba un largo superior o igual a dos veces el diámetro de la semilla. Finalizado los 40 días se realizó un test de corte a las semillas no germinadas para separar las semillas visualmente sanas de las probablemente no viables, que presentaban su contenido calcinado o con un daño evidente.

\section{Análisis estadísticos}

Se utilizó un ANDEVA de dos factores para examinar el efecto de la carga de combustible y la profundidad en la temperatura máxima registrada al interior del banco de semillas. El porcentaje de germinación de semillas de $T$. monspessulana a diferentes temperaturas en condiciones de laboratorio fue analizado con un ANDEVA. Pare ello, los datos de germinación fueron transformados a Arcsen $\% \mathrm{G}^{1 / 2}$ para obtener una distribución normal y cada tiempo de exposición a las temperaturas fue analizado de forma independiente. La prueba de Tukey fue utilizada para detectar las temperaturas significativamente distintas entre si. La respuesta de T. monspessulana a los diferentes tratamientos de quema se evaluó mediante una ANDEVA con un diseño en bloques en donde cada población se consideró un bloque. Los datos de germinación también fueron transformados a Arcsen $\% \mathrm{G}^{1 / 2}$ para obtener una distribución normal.

\section{RESULTADOS}

\section{Respuesta del banco de semillas al fuego}

En las muestras de los tratamientos de quema baja y quema alta la temperatura máxima promedio a nivel superficial se registró entre los 8 y 15 minutos, dependiendo de la carga de combustible, y entre 18 y 30 minutos a $2 \mathrm{~cm}$ de profundidad (Fig. 1). La carga de combustible influye significativamente en la temperatura $\left(\mathrm{F}_{1,41}=6.58 ; \mathrm{P}=0.0141\right)$. Así, con una carga de combustible equivalente a 5 ton $\mathrm{ha}^{-1}$ la temperatura máxima promedio $( \pm \mathrm{EE})$ registrada al interior del banco fue de $110 \pm 19$ ${ }^{\circ} \mathrm{C}(\mathrm{n}=22)$ y de $178 \pm 27{ }^{\circ} \mathrm{C}(\mathrm{n}=24)$ con una carga de combustible equivalente a 10 ton $\mathrm{ha}^{-1}$ (Fig. 2). La profundidad a la que estén las semillas en el suelo también influye significativamente en la temperatura a que estas pueden estar expuestas $\left(\mathrm{F}_{1,41}=28.4 ; \mathrm{P}<\right.$ $0.0001)$. Superficialmente la temperatura máxima promedio $\left( \pm \mathrm{EE}\right.$ ) fue de $212 \pm 25^{\circ} \mathrm{C}$ ( $\mathrm{n}$ $=23)$ y a $2 \mathrm{~cm}$ de profundidad fue de $73 \pm 11^{\circ} \mathrm{C}$ $(\mathrm{n}=23)$. La interacción entre ambos factores no fue significativa $\left(\mathrm{F}_{1,41}=0.2 ; \mathrm{P}=0.5234\right)$.

El número promedio de semillas $( \pm \mathrm{EE})$ almacenadas en el banco entre ambos sitios fue de $8703 \pm 3434$ semillas $\mathrm{m}^{-2}(\mathrm{n}=36)$. La germinación final no fue distinta entre ambas poblaciones $\left(\mathrm{F}_{1,23}=0.07 ; \mathrm{P}=0.7962\right)$. Las semillas de las muestras sometidas al fuego, tratamientos quema baja y quema alta, alcanzaron una germinación promedio $( \pm \mathrm{EE})$ de $46.7 \% \pm 6.7$ y de $45.6 \% \pm 7.2$, respectivamente, siendo significativamente mayores al tratamiento sin quema $\left(\mathrm{F}_{2,23}=10.4\right.$; $\mathrm{P}=0.0006)$ que solo registró un $11.4 \% \pm 2.8 \mathrm{de}$ germinación (Fig. 3). 


\section{Temperaturas óptimas para la germinación}

En aquellos tratamientos en los que se registró germinación, esta comenzó entre el día 13 y el 19 tras la instalación del ensayo. En términos generales los tratamientos entre 90 y $130{ }^{\circ} \mathrm{C}$ para 5 minutos y entre 90 y $110{ }^{\circ} \mathrm{C}$ para 10 minutos, mostraron un porcentaje de germinación superior a las semillas sin tratamiento de temperatura, las que alcanzaron una germinación de $2.5 \%$. La germinación máxima de las semillas de $T$. monspessulana cuando son calentadas por 10 minutos ocurre entre 80 y $120{ }^{\circ} \mathrm{C}$, alcanzando un $28.8 \%$ (Tabla

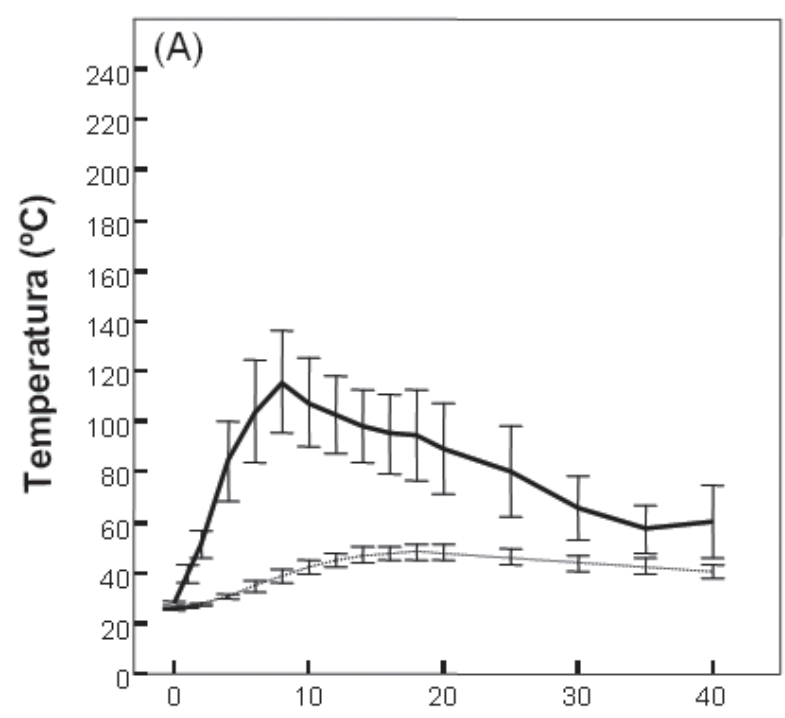

Tiempo (min)

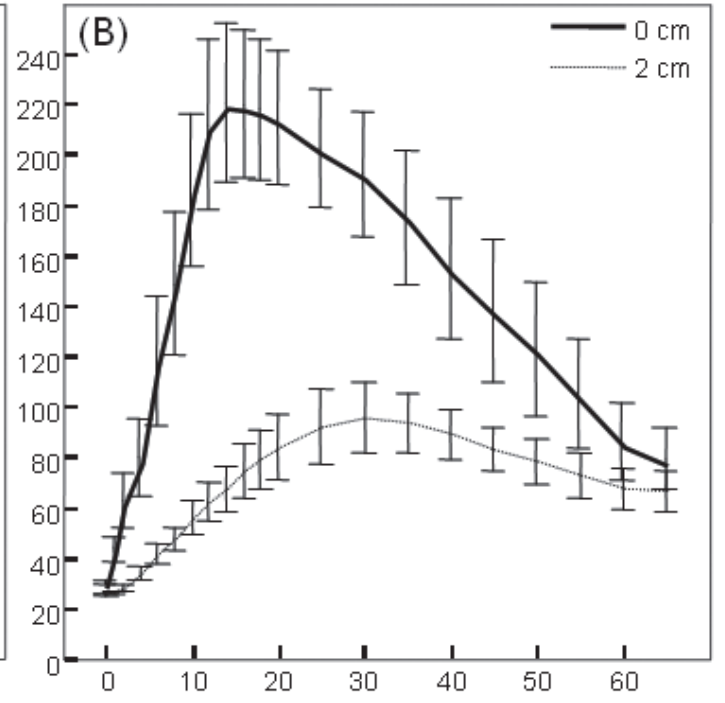

Tiempo ( $\mathrm{min})$

Fig. 1: Temperatura máxima promedio $( \pm \mathrm{EE})$ del suelo a 0 y $2 \mathrm{~cm}$ de profundidad bajo distintas cargas de combustibles A) 5 ton ha- ${ }^{-1}$ B) 10 ton ha- ${ }^{-1}$.

Maximum average temperature $( \pm \mathrm{SE})$ of the soil at 0 and $2 \mathrm{~cm}$ of depth exposed to different fuel load A) 5 ton ha- ${ }^{-1}$ B) 10 ton $\mathrm{ha}^{-1}$.

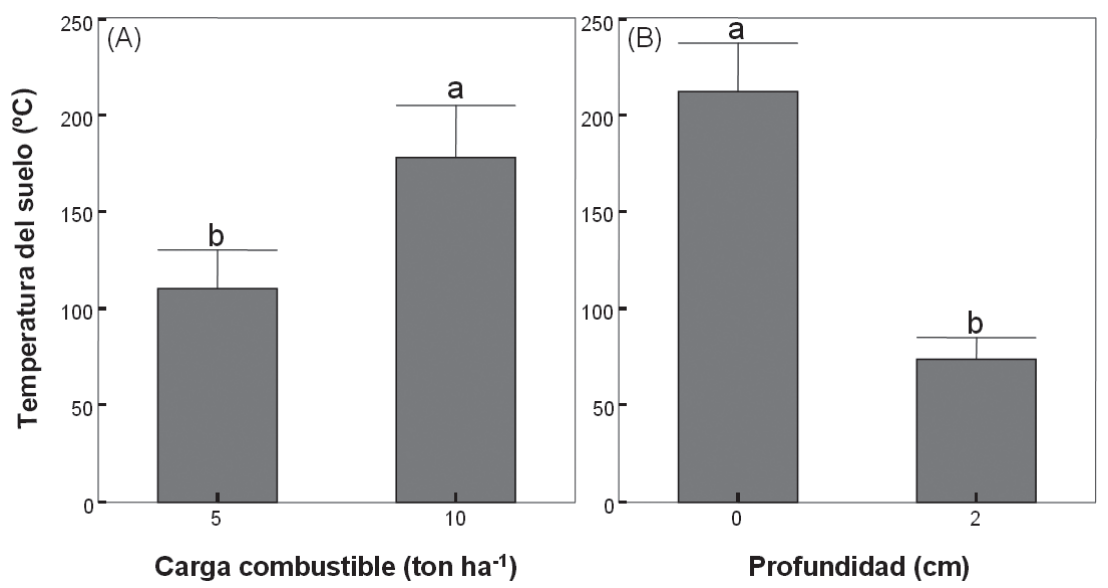

Fig. 2: Temperatura máxima promedio ( \pm EE) alcanzada por el banco de semillas. A) con dos cargas de combustible distintas. B) a distintas profundidades. Letras distintas indican diferencias significativas entre tratamientos (Prueba de Tukey, $\mathrm{P}<0.005$ ).

Maximun average temperature $( \pm \mathrm{SE})$ reached by the seed bank. A) under two different fuel loads. B) at different depths. Different letters show significant differences between treatments (Tukey's Test, $\mathrm{P}<0.005$ ). 
1). La exposición al calor por 5 minutos generó una germinación máxima de $26.3 \%$, no variando significativamente entre los 80 y 130 ${ }^{\circ} \mathrm{C}$, observándose una alta variabilidad en este rango. Además, se observó un marcado aumento en la cantidad de semillas calcinadas a partir de los 120 y $130{ }^{\circ} \mathrm{C}$, llegando a la totalidad de las semillas por sobre los $140{ }^{\circ} \mathrm{C}$ (Tabla 1). A temperaturas inferiores de $120^{\circ} \mathrm{C}$ las semillas no germinadas se mantuvieron mayoritariamente sanas.

\section{DISCUSIÓN}

La quema de hojarasca estimuló la germinación de semillas de $T$. monspessulana desde el banco de semillas, tanto en condiciones de alta como de baja carga de combustible. Este efecto estimulador del fuego en la germinación de T. monspessulana se debería a que sus semillas poseen una testa altamente cutinizada y muy lisa que les proporciona una latencia exógena fuerte, $\mathrm{y}$ ha conducido al término de "semillas duras"

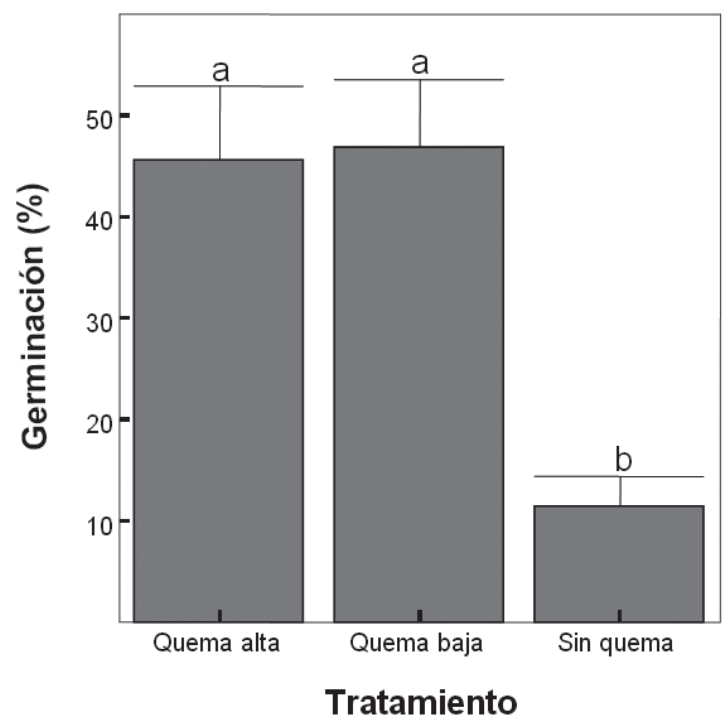

Fig. 3: Germinación promedio de semillas de $T$. monspessulana $( \pm \mathrm{EE})$ sometidas a diferentes tratamientos de quema después de 60 días. Letras distintas indican diferencias significativas entre tratamientos (Prueba de Tukey, $\mathrm{P}<0.005)$.

Mean germination of $T$. monspessulana seeds $( \pm \mathrm{SE})$ under different burn treatments after 60 days. Diferent letters show significant differences between treatments (Tukey's Test, $\mathrm{P}<0.005)$.
(Baskin \& Baskin 1998, Keeley \& Fotheringhan 2000). Esta barrera en la testa de la semilla impide el paso de agua y oxígeno durante el proceso de imbibición (Baskin \& Baskin 1998). Esta condición hace que la germinación de las semillas de $T$. monspessulana sea baja en condiciones normales, pero de acuerdo a los resultados obtenidos, esta aumentaría sus niveles de germinación al ser sometidas a altas temperaturas. Es conocido que las altas temperaturas generan fracturas en las testas de semillas duras por donde puede pasar el agua y el oxígeno durante la imbibición, permitiendo así la germinación del embrión (Baskin \& Baskin 1998).

Entre las especies de semillas duras existe una gran variación en la respuesta de las semillas a distintas intensidades y duraciones del calor, así como también en las temperaturas óptimas de germinación. Algunas especies tienen su óptimo de germinación con exposiciones breves a altas temperaturas $\left(105{ }^{\circ} \mathrm{C}\right.$ por 5 minutos), mientras que otras la logran a temperaturas más bajas, pero por un largo tiempo $\left(70{ }^{\circ} \mathrm{C}\right.$ por 1 hora $)$ (Keeley \& Fotheringhan 2000). En el caso de T. monspessulana, la máxima germinación se registró entre los 80 y 130 independiente del tiempo de exposición. Estos resultados podrían sugerir que el efecto estimulador del fuego en $T$. monspessulana ocurriría en una amplia ventana de combinaciones de altas temperaturas y tiempos de exposición a ellas. Sin embargo, con el aumento excesivo de las temperaturas la posibilidad de sobrevivencia de las semillas se ve afectada negativamente (Banda et al. 2006). En la mayoría de las especies, tiempos de exposición mayores a 5 minutos entre 120 y $150{ }^{\circ} \mathrm{C}$ causan la muerte de las semillas o reducen significativamente su germinación (e.g., Mallik \& Giminghan 1985, Tarrega et al. 1992, Herranz et al. 1998, Núñez et al. 2003, Williams et al. 2003, Alvarez et al. 2007, Herrero et al. 2007). Las semillas de $T$. monspessulana seguirían este mismo patrón, experimentando una brusca caída en su germinación desde los $120{ }^{\circ} \mathrm{C}$ por 10 minutos y la muerte de todas ellas desde los $140{ }^{\circ} \mathrm{C}$. Por lo tanto, nuestros resultados sugieren una pequeña ventana entre las temperaturas que estimulan la germinación y aquellas que resultan ser letales para esta especie invasora. 
La distribución vertical de las semillas al interior del banco juega un rol fundamental para asegurar la supervivencia de las semillas después de un incendio. La mayor cantidad de propágulos de los bancos de semillas se encuentra en los primeros $10 \mathrm{~cm}$ de suelo (e.g., Williams 1981, Muñoz \& Fuentes 1989, Alexander \& D’Antonio 2003a, Auld \& Denham 2006), sugiriendo una ventaja ecológica importante para aquellas especies presentes en el banco de semillas y cuya germinación es estimulada por el fuego. Sin embargo, la ocurrencia de un incendio no asegura la estimulación de las semillas ya que muchas veces los incendios causan efectos inesperados e irregulares en el suelo, dependiendo tanto de las características y acumulación del combustible, así como de las propiedades físicas del suelo (DeBano et al. 2005). Además la transferencia calórica de un incendio forestal al suelo es relativamente baja registrándose temperaturas menores a $60{ }^{\circ} \mathrm{C}$ entre $\operatorname{los} 2$ y $5 \mathrm{~cm}$ de profundidad (Raison et al. 1986, Valette et al. 1994, Bradstock \& Auld 1995, Penman \& Towerton 2008). Más aún, la temperatura del suelo estará determinada por la carga de combustible y la humedad del suelo, así, cuando la carga de combustible es alta y el suelo está seco se pueden registrar temperaturas superiores a $330{ }^{\circ} \mathrm{C}$ a $2 \mathrm{~m}$ de profundidad (Behenna et al. 2008). Auld \& Denham (2006) señalan que después de un fuego muy pocas semillas vivas se encuentran en los primeros $5 \mathrm{~cm}$ de suelo y que el fuego, bajo esa profundidad, reduce su capacidad para romper la latencia de las semillas. Penman \& Towerton (2008) afirman que entre 2 y $5 \mathrm{~cm}$ de profundidad rara vez es posible registrar temperaturas que induzcan la germinación de especies adaptadas a incendios forestales. En consecuencia, para $T$. monspessulana la porción de suelo que alcanza las temperaturas y tiempos óptimos para estimular la germinación de semillas es muy pequeña ocurriendo solo en áreas con una carga de combustible relativamente baja o bien en una delgada franja de suelo no superior a 1 $\mathrm{cm}$ de ancho, ya que en la superficie las temperaturas resultan letales $\sim 210^{\circ} \mathrm{C}$ y a $2 \mathrm{~cm}$ de profundidad no superan $\operatorname{los} 80^{\circ} \mathrm{C}$.

A pesar de las limitaciones anteriormente mencionadas, $T$. monspessulana tendría una ventaja adicional en Chile debido a que la mayoría de las especies nativas no estarían adaptadas al fuego. Las especies cuya germinación es estimulada por el calor son

TABLA 1

Porcentaje de semillas germinadas $( \pm \mathrm{EE})$, semillas no germinadas y semillas calcinadas al final del ensayo para cada combinación de temperatura y tiempo. Letras distintas indican diferencias significativas entre tratamientos (Prueba de Tukey, $\mathrm{P}<0.005$ ).

Percentage of germinated seeds $( \pm \mathrm{SE})$, not germinated seeds and calcined seeds at the end of the experiment for each combination of temperature and time. Diferent letters show significant differences between treatments (Tukey's Test , $\mathrm{P}<0.005)$.

\begin{tabular}{|c|c|c|c|c|c|c|c|c|c|c|}
\hline \multirow{3}{*}{$\begin{array}{l}\text { Temperatura } \\
\left({ }^{\circ} \mathrm{C}\right) \\
\text { Control }\end{array}$} & \multicolumn{4}{|c|}{ Germinación (\%) 5 minutos } & \multicolumn{6}{|c|}{ Germinación (\%) 10 minutos } \\
\hline & Germinadas & No germinadas & \multicolumn{2}{|c|}{ Calcinadas } & \multicolumn{2}{|c|}{ Germinadas } & \multicolumn{2}{|c|}{ No germinadas } & \multicolumn{2}{|l|}{ Calcinadas } \\
\hline & $2.5 \pm 2.9$ & $96.3 \pm 2.5$ & $1.3 \pm 2.5$ & $\mathrm{c}$ & $2.5 \pm 2.9$ & $\mathrm{bc}$ & $96.3 \pm 2.5$ & a & $1.3 \pm 2.5$ & $\mathrm{~d}$ \\
\hline 80 & $11.3 \pm 9.5$ & $87.5 \pm 8.7$ & $1.3 \pm 2.5$ & $\mathrm{c}$ & $11.3 \pm 4.8$ & $a b$ & $87.5 \pm 6.5$ & $\mathrm{ab}$ & $1.3 \pm 2.5$ & $\mathrm{~d}$ \\
\hline 90 & $26.3 \pm 21.8$ & $67.5 \pm 16.6 \mathrm{bc}$ & $6.3 \pm 6.3$ & $\mathrm{c}$ & $25.0 \pm 10.8$ & a & $73.8 \pm 12.5$ & $a b$ & $1.3 \pm 2.5$ & $\mathrm{~d}$ \\
\hline 100 & $20.0 \pm 4.1$ & $78.8 \pm 4.8$ & $1.3 \pm 2.5$ & $\mathrm{c}$ & $28.8 \pm 4.8$ & $\mathrm{a}$ & $66.3 \pm 4.8$ & $\mathrm{bc}$ & $5.0 \pm 0.0$ & $\mathrm{~cd}$ \\
\hline 110 & $22.5 \pm 2.9$ & $77.5 \pm 2.9 \quad \mathrm{bc}$ & $0.0 \pm 0.0$ & $\mathrm{c}$ & $28.8 \pm 19.3$ & $\mathrm{a}$ & $53.8 \pm 17.5$ & $\mathrm{c}$ & $17.5 \pm 6.5$ & $\mathrm{c}$ \\
\hline 120 & $26.3 \pm 4.8$ & $65.0 \pm 7.1$ & $8.8 \pm 4.8$ & $\mathrm{c}$ & $11.3 \pm 6.3$ & $a b$ & $8.8 \pm 14.4$ & $\mathrm{~d}$ & $80.0 \pm 14.7$ & b \\
\hline 130 & $23.8 \pm 18.0$ & $11.3 \pm 7.5$ & $65.0 \pm 22.7$ & b & $3.8 \pm 2.5$ & bc & $5.0 \pm 5.8$ & $\mathrm{~d}$ & $91.3 \pm 4.8$ & b \\
\hline 140 & $5.0 \pm 7.1$ & $0.0 \pm 0.0$ & $95.0 \pm 7.1$ & $\mathrm{a}$ & $0.0 \pm 0.0$ & $\mathrm{c}$ & $0.0 \pm 0.0$ & d & $100.0 \pm 0.0$ & $\mathrm{a}$ \\
\hline 150 & $0.0 \pm 0.0$ & $2.5 \pm 5.0$ & $97.5 \pm 5.0$ & $a$ & $0.0 \pm 0.0$ & $\mathrm{c}$ & $0.0 \pm 0.0$ & d & $100.0 \pm 0.0$ & $\mathrm{a}$ \\
\hline 160 & $0.0 \pm 0.0$ & $2.5 \pm 5.0$ & $97.5 \pm 5.0$ & $\mathrm{a}$ & $0.0 \pm 0.0$ & $\mathrm{c}$ & $1.3 \pm 2.5$ & $d$ & $98.8 \pm 2.5$ & $\mathrm{a}$ \\
\hline 170 & $0.0 \pm 0.0$ & $0.0 \pm 0.0$ & $100.0 \pm 0.0$ & $\mathrm{a}$ & $0.0 \pm 0.0$ & $\mathrm{c}$ & $0.0 \pm 0.0$ & d & $100.0 \pm 0.0$ & $\mathrm{a}$ \\
\hline 180 & $0.0 \pm 0.0$ & $0.0 \pm 0.0$ & $100.0 \pm 0.0$ & $\mathrm{a}$ & $0.0 \pm 0.0$ & $\mathrm{c}$ & $0.0 \pm 0.0$ & d & $100.0 \pm 0.0$ & $\mathrm{a}$ \\
\hline
\end{tabular}


frecuentes en todas las zonas de matorral de clima mediterráneo que están regularmente expuestas a la renovación de la vegetación por parte del fuego (Keeley \& Fotheringhan 2000). Sin embargo, en el caso de la mayoría de las semillas de los arbustos nativos del matorral chileno la estimulación directa del fuego no estaría presente (Muñoz \& Fuentes 1989, Gómez-González \& Cavieres 2009) así como tampoco una estimulación indirecta a través del humo generado en los incendios (GómezGonzález et al. 2008). Por otro lado, actualmente un importante número de especies introducidas estimuladas por el fuego se encuentran creciendo en el territorio nacional, entre ellas $T$. monspessulana (Figueroa et al. 2004, Pauchard et al. 2004, García et al. 2007). La probabilidad de que áreas con presencia de T. monspessulana se vean afectadas por un incendio forestal es relativamente alta, principalmente en la Región del Biobío que es la que concentra la mayor cantidad de incendios forestales a nivel nacional (Peña \& Valenzuela 2008) y una importante abundancia de T. monspessulana (Matthei 1995, García et al. 2007, Pauchard et al. 2008). Todo esto podría llevar a un cambio importante en la composición de especies en aquellas zonas bajo la constante amenaza del fuego y con presencia de especies introducidas.

La estrategia de $T$. monspessulana de generar bancos de semillas superiores a 8000 semillas $\mathrm{m}^{-2}$, compensaría en parte la heterogeneidad del efecto del fuego en el suelo y la estrecha ventana de temperaturas capaces de estimular la germinación de las semillas. La capacidad de esta especie de producir semillas entre los dos y tres años (Bossard 2000, Parsons \& Cuthbertson 2001) le daría otra ventaja competitiva frente a la acción del fuego en zonas donde el periodo de retorno de incendios de magnitud varía entre 9 y 17 años (Peña \& Valenzuela 2008).

Finalmente, podemos concluir que las semillas de $T$. monspessulana aumentan su germinación con las altas temperaturas y que los incendios forestales, pueden generar condiciones térmicas en los primeros centímetros del suelo suficientes para estimular la germinación de la especie. El éxito de esta especie se basaría principalmente en su capacidad de formar grandes bancos de semillas, aumentando la probabilidad de que las semillas sobrevivan y sean estimuladas por el fuego. Después de un incendio forestal $T$. monspessulana presenta un rápido crecimiento en altura (García et al. 2007), llegando a acumular una biomasa en estado adulto cercana a las 80 ton ha-1 (García et al., datos no publicados) de fácil ignición dada la gran cantidad de ramillas de diámetro inferior a 5 $\mathrm{mm}$. Así, los eventos de fuego están incentivando la renovación de las poblaciones de T. monspessulana con nuevas y vigorosas plantas provenientes del banco de semillas.

La confirmación de la interacción positiva entre el fuego y la germinación de las semillas de T. monspessulana, nos permite recomendar una modificación fundamental a ciertas prácticas habituales, como la quema de los desechos resultantes de la cosecha en plantaciones forestales de $P$. radiata. También, a ciertas técnicas de manejo para la vegetación no deseada en cultivos (agrícolas y forestales) y otras áreas, en donde es habitual realizar quemas para "controlar" o eliminar malezas o plantas invasoras. Estas prácticas, más que ayudar a la erradicación podría estar favoreciendo la permanecía de $T$. monspessulana en el sistema, así como la de otras especies de la misma familia presentes en el país con un comportamiento probablemente similar, como Ulex europaeus L., Cytisus scoparius L. (Link) (e.g., Rivas et al. 2006, Hanley 2009) y algunas especies de Acacia. Si bien la realización de quemas de alta intensidad podrían asegurar la muerte de las semillas de $T$. monspessulana, las pérdidas por volatilización de nutrientes (Knoepp et al. 2005) y la muerte de la microflora y microfauna del suelo (Busse \& DeBano 2005) pueden causar perjuicios mayores a la permanencia de la especie. Siendo recomendable evitar estas prácticas en zonas con presencia de T. monspessulana. Por otro lado, el escenario de invasión de especies de Fabaceae se presenta bastante complejo si se considera el patrón actual de aumento de incendios forestales en la zona centro-sur de Chile. Lo más probable es que la invasión de estas especies aumente afectando incluso aquellos fragmentos de bosques nativos que sobreviven en la matriz dominada por plantaciones forestales, por lo que deben considerarse otras alternativas que permitan disminuir la expansión de estas especies y fomentar la conservación y restauración de la vegetación nativa remanente. 


\section{AGRADECIMIENTOS}

Financiado por FONDECYT 1070488, ICM P05-002 y CONICYT PFB-023. RA García es financiado por Beca de Estudios de Doctorado CONICYT. Esta investigación es una contribución del Laboratorio de Invasiones Biológicas (LIB), IEB-UdeC.

\section{LITERATURA CITADA}

ADAMS R \& D SIMMONS (1991) The invasive potential of Genista monspessulana (Montpellier broom) in dry sclerophyll forest in Victoria. Victorian Naturalist 108: 84-89.

ALEXANDER J \& C D'ANTONIO (2003a) Seed bank dynamics of French broom in coastal California grasslands: Effects of stand age and prescribed burning on control and restoration. Restoration Ecology 11: 185-197.

ALEXANDER J \& C D'ANTONIO (2003b) Control methods the removal of French and Scotch Broom tested in coastal California. Ecological Restoration 21: 191-198.

ALVAREZ R, L VALBUENA \& L CALVO (2007) Effect of high temperatures on seed germination and seedling survival in three pine species (Pinus pinaster, $P$. Sylvestris and $P$. nigra). International Journal of Wildland Fire 16: 63-70.

ARROYO MTK, CM MARTICORENA, O MATTHEI \& L CAVIERES (2000) Plant invasions in Chile: Present patterns and future predictions. En: Mooney HA \& RJ Hobbs (eds) Invasive species in a changing world: 385-421. Island Press, Washington DC.

AULD T \& A DENHAM (2006) How much seed remains in the soil after a fire? Plant Ecology 187: $15-24$.

BANDA T, MW SCHWARTZ, \& T CARO (2006) Effects of fire on germination of Pterocarpus angolesis. Forest Ecology and Management 233: 116-120.

BASKIN CC \& JM BASKIN (1998) Seeds. Ecology, biogeography, and evolution of dormancy and germination. Academic Press, San Diego, CA.

BEHENNA M, S VETTER \& S FOURIE (2008) Viability of alien and native seed bank after slash and burn: Effets of soil moisture, depth of burial and fuel load. South African Journal of Botany 74: 454-462.

BOSSARD C (2000) Genista monspessulana (L.) L. Johnson. En: Bossard CC, JM Randall \& MC Hoshovsky (eds) Invasive plants of California's wildlands: 203-208. University of California Press, Berkeley, USA.

BRADSTOCK RA \& TD AULD (1995) Soil temperatures during experimental bushfires in relation to fire intensity: Consequences for legume germination and fire management in south-eastern Australia. Journal of Applied Ecology 32: 76-84.

BROOKS ML, CM D'ANTONIO, DM RICHARDSON, JB GRACE \& JE KEELEY (2004) Effects of invasive plants on fire regimes. BioScience 54: 677-688.

BUSSE MD \& LF DEBANO (2005) Soil Biology. En: Neary DG, KC Ryan \& LF DeBano (eds) Wildland fire in ecosystems: Effects of fire on soils and water: 73-91. U.S. Department of
Agriculture, Forest Service, Rocky Mountain Research Station, Colorado, USA.

COCHRANE M \& C BARBER (2008) Climate change, human land use y future fires in the amazon. Global Change Biology 15: 601-612.

CONAF (2009) Estadísticas de incendios. Corporación Nacional Forestal, Chile. URL: http:// w w w. c o n a f.c l/? ? p a g e = ho m e / contents\&seccion_id=322af25bd24d696f5abe1ed $700 \mathrm{dc} 5828 \&$ unidad $=8 \&$ pagina $=$ (accedido Marzo 17, 2009).

CRAMER W (2001) Fire ecology, Mediterranean forest and global change. Forest Ecology and Management 147: 1-2.

D’ANTONIO CM (2000) Fire, plant invasions, and global changes. En: Mooney HA \& RJ Hobbs (eds) Invasive species in a changing world: 6595. Ireland Press, Covelo, California, USA.

D'ANTONIO CM \& PM VITOUSEK (1992) Biological invasions by exotic grasses, the grass/fire cycle, and global change. Annual Review of Ecology and Systematics 23: 63-87.

DEBANO LF, NEARY DG \& PF FFOLLIOTT (2005) Soil biology. En: Neary DG, KC Ryan \& LF DeBano (eds) Wildland fire in ecosystems: Effects of fire on soils and water: 73-91. U.S. Department of Agriculture, Forest Service, Rocky Mountain Research Station, Colorado, USA.

DI CASTRI F (1991) An ecological overview of the five regions of the world with Mediterranean climate. En: Groves RH \& F Di Castri (eds) Biogeography of Mediterranean invasions: 3-15. Cambridge University Press, Cambridge, UK.

FIGUEROA JA, SA CASTRO, PA MARQUET \& FM JACKSIC (2004) Exotic plants invasions to the mediterranean región of Chile: Causes, history and impacts. Revista Chilena de Historia Natural 77: 465-483.

FIGUEROA JA, LA CAVIERES, S GÓMEZ-GONZÁLEZ, MA MOLINA-MONTENEGRO \& FM JAKSIC (2009) Do heat and smoke increase emergence of exotic and native plants in the matorral of central Chile? Acta Oecologica 35: 335-340.

GARCÍA RA, A PAUCHARD \& E PEÑA (2007) Invasión de Teline monspessulana (L.) K. Koch después de un incendio forestal: Banco de semillas, regeneración y crecimiento. Gayana Botánica 64: 200-209.

GÓMEZ-GONZÁLEZ S \& L CAVIERES (2009) Litter burning does not equally affect emergence on native and alien species of the mediterraneantype Chilean matorral. International Journal of Wildland Fire 18: 213-221.

GÓMEZ-GONZÁLEZ S, A SIERRA-ALMEIDA \& L CAVIERES (2008) Does plant-derived smoke affect seed germination in dominant woody species of the Mediterranean matorral of central Chile? Forest Ecology and Management 255: 1510-1515.

HANLEY ME (2009) Thermal shock and germination in North-West European Genisteae: Implications for heathland management and invasive weed control using fire. Applied Vegetation Science 12: $385-390$.

HERRANZ J, P FERRANDIS \& J MARTÍNEZSÁNCHEZ (1998) Influence of heat on seed germination of seven Mediterranean Leguminosae species. Plant Ecology 136: 95-103.

HERRERO C, R SAN MARTIN \& F BRAVO (2007) 
Effect of heat and ash treatments on germination of Pinus pinaster and Cistus laurifolius. Journal of Arid Environments 70: 540-548.

IPCC (2007) Resumen para responsables de políticas. En: Parry ML, OF Canziani, JP Palutikof, PJ van der Linden \& CE Hanson (eds) Cambio climático 2007: Impactos y vulnerabilidad: 2-23. Cambridge University Press, Cambridge, UK.

JOHNSON PH (1982) Naturalized plants in Southwest South. Island, New Zealand. New Zealand Journal of Botany 20: 131-142.

KEELEY JE, D LUBIN \& CJ FOTHERINGHAM (2003) Fire and grazing impacts on plant diversity and alien plant invasions in the southern Sierra Nevada. Ecological Applications 13: 1355-1374.

KEELEY JE \& CJ FOTHERINGHAM (2000) Role of fire in regeneration from seed. En: Fenner M (ed) Seeds: The ecology of regeneration in plant communities: 311-330. Segunda edición. CAB International, Oxon, UK.

KNOEPP JD, LF DEBANO \& DG NEARY (2005) Soil chemestry. En: Neary DG, KC Ryan \& LF DeBano (eds) Wildland fire in ecosystems: Effects of fire on soils and water: 73-91. U.S. Department of Agriculture, Forest Service, Rocky Mountain Research Station, Colorado, USA.

MACK MC \& CM D'ANTONIO (1998) Impacts of biological invasions on disturbance regimes. Trends in Ecology and Evolution 13: 195-198.

MACK RN, D SIMBERLOFF, WM LONSDALE, H EVANS, M CLOUT \& F BAZZAZ (2000) Biotic invasions: Causes, epidemiology, global consequences and control. Ecological Applications 10: 689-710.

MALLIK AU \& CH GIMINGHAN (1985) Ecological efect of heater burning. II. Efects on seed germination and vegetative regeneration. Journal of Ecology 73: 633-644.

MATTHEI O (1995) Manual de las malezas que crecen en Chile. Alfabeta Impresores, Santiago, Chile.

MUÑOZ MR \& ER FUENTES (1989) Does fire induce shrub germination in the Chilean matorral. Oikos 56: 177-181.

NLWRA (2008) The distribution of some significant invasive plants in Australia 2007. National Land and Water Resources Audit, Canberra, Australia.

NUÑEZ Ma R, F BRAVO \& L CALVO (2003) Predecting the probabilty of seed germination in Pinus sylvestris L. and four competitor shrub species after fire. Annals of Forest Science 60: 75-81.

PARSONS WT \& EG CUTHBERTSON (2001) Noxious weeds of Australia. Segunda edición. CSIRO Publishing, Collingwood, Victoria, Australia.

PAUCHARD A, L CAVIERES, R BUSTAMANTE, P BECERRA \& E RAPOPORT (2004) Increasing the understanding of plant invasions in southern South America: First symposium on alien plant invasions in Chile. Biological Invasions 6: 255257.

PAUCHARD A \& P ALABACK (2002) La amenaza de plantas invasoras. Chile Forestal 289: 13-15.

PAUCHARD A \& P ALABACK (2004) Influence of elevation, land use, and landscape context on patterns of alien plant invasions along roadsides in protected areas of south-central Chile. Conservation Biology 18: 238-248.

PAUCHARD A, RA GARCIA, E PEÑA, C GONZÁLEZ, LA CAVIERES \& R BUSTAMANTE (2008) Positive feedbacks between plant invasions and fire regimes: Teline monspessulana (L.) K.Koch (Fabaceae) in central Chile. Biological Invasions 10: $547-553$.

PENMAN TD \& AL TOWERTON (2008) Soil temperatures during autumn prescribed burning: Implications for the germination of fire responsive species? International Journal of Wildland Fire 17: 572-578

PEÑA E \& L VALENZUELA (2008) Incremento de los incendios forestales en bosques naturales y plantaciones forestales en Chile. En: GonzálezCabán A (ed) Memorias del segundo simposio internacional sobre políticas, planificación y economía de los programas de protección contra incendios forestales: Una visión global: 595-612. Gen. Tech. Rep. PSW-GTR-208. Departamento de Agricultura de los EE.UU., Servicio Forestal, Estación de Investigación del Pacífico Suroeste, Albany, California.

RAISON RJ, PV WOODS, BF JAKOBSEN \& GAV BARY (1986) Soil temperatures during and following low-intensity prescribed burning in a Eucalyptus pauciflora forest. Australian Journal of Soil Research 24: 33-47.

RIVAS M, O REYES \& M CASAL (2006) Influence of heat and smoke treatments on the germination of six leguminous shrubby species. International Journal of Wildland Fire 15: 73-80.

SMITH JMB (1994) The changing ecological impact of broom (Cytisus scoparius) at Barrington Tops, New South Wales. Plant Protection Quarterly 9: 6-11.

STOHLGREN TJ, Y OTSUKI, CA VILLA, M LEE \& J BELNAP (2001) Patterns of plant invasions: A case example in native species hotspots and rare habitats. Biological Invasions 3: 37-50.

TARREGA R, L CALVO \& L TRABAUD (1992) Effect of high temperatures on seed germination of two woody Leguminosae. Vegetatio 102: 139-147.

VALETTE JC, V GOMENDY, J MARÉCHAL, C HOUSSARD \& D GILLON (1994) Heat transfer in the soil during very low-intensity experimental fires: The role of duff and soil moisture content. International Journal of Wildland Fire 4: 225-237.

WATERHOUSE BM (1998) Broom (Cytisus scoparius) at Barrington Tops, New South Wales. Australian Geographical Studies 26: 239-248.

WILLIAMS PA (1981) Aspects of the ecology of broom (Cytisus scoparius) in Canterbury, New Zaeland. New Zaeland Journal of Botany 19: 31-43.

WILLIAMS PR, RA CONGDON, AC GRICE \& PJ CLARKE (2003) Fire-related cues break seed dormancy of six legumes of tropical eucalypt savannas in north-eastern Australia. Austral Ecology 28: 507-514.

ZOUHAR K (2005) Genista monspessulana. En: Fire Effects Information System. U.S. Department of Agriculture, Forest Service, Rocky Mountain Research Station, Fire Sciences Laboratory (Producer). URL: http://www.fs.fed.us/ database/feis/ (accedido Julio 20, 2009). 